\title{
REVISIONES
}

\section{Configuración activa de la identidad en la escuela como un espacio-tiempo}

\author{
Active configuration of identity in the school as a space-time \\ Configuração ativa da identidade na escola como um espaço-tempo
}

\begin{abstract}
Bastián Andrés Moreno Molina
Estudiante de Magíster en Neurociencias aplicadas a la Educación, Universidad Finis Terrae, Santiago, Chile. Teléfono: (56) 996954515 . Correo electrónico: bastianmorenom@gmail.com
\end{abstract}

\section{RESUMEN}

Este trabajo pretende ser un aporte a la reflexión de las experiencias educativas apuntando a una mirada de la condición biológica de autonomía de los seres humanos como agentes cognitivos situados, es decir, se plantea un enfoque biológico y epistemológico que concibe al conocimiento como configurado desde una matriz ecológica histórica y permitido por el sostenimiento activo de la identidad del individuo. Estas nociones se acompañan con la creación de un corpus de conocimiento en la escuela que describe las características del espacio-tiempo concreto. Como consecuencia, es una invitación a repensar nuestra mirada de lo humano y del fenómeno del aprendizaje escolar.

Palabras clave: escuela, aprendizaje, autonomía, identidad, conocimiento escolar.

\section{ABSTRACT}

This paper aims to contribute to the reflection of the educational experiences pointing to an approach of the biological condition of autonomy of human beings as situated cognitive agents, that is, to suggest a biological and epistemological approach which understands knowledge as configured from a historical ecological matrix and permitted by the active maintenance of the individual identity. These notions are accompanied by the creation of a corpus of knowledge in school describing the concrete space-time characteristics. As consequence, it is an invitation to rethink our view of the human and the phenomenon of school learning.

Key words: school, learning, autonomy, identity, school knowledge.

\section{RESUMO}

Pretende-se contribuir com a reflexão das experiências educativas, indicando um novo olhar da condição biológica de autonomia dos seres humanos como agentes cognitivos situados, ou seja, expõe-se um enfoque biológico e epistemológico que concebe o conhecimento como aquele configurado desde uma matriz ecológica histórica e permitido pela sustentação ativa da identidade do sujeito. Estas noções são acompanhadas pela criação de um corpus de conhecimento que descreve as características do espaço-tempo concreto da escola. Consequentemente, é um convite a repensar nossa visão do humano e do fenômeno da aprendizagem escolar.

Palavras Chave: escola, aprendizagem, autonomia, identidade, conhecimento escolar. 


\section{INTRODUCCIÓN}

La escuela se constituye permanentemente como un espacio-tiempo, forjando en su historia particular una identidad cultural que la caracteriza como tal. Esta permanente construcción, como un continuo hacerse, está guiada por el fluir de las conversaciones que sus miembros viven en su estar allí. De esta forma sus participantes se transforman en un devenir propio del tipo de relaciones que se gesten en las interacciones del espacio común. En este sentido la escuela no es un espacio dado, no es un tiempo predeterminado, aunque las acciones de sus dirigentes administrativos intenten controlarlo, la escuela es más bien un espacio-tiempo que surge momento a momento y por tanto que tiene ese efecto en sus miembros. Al ser, en cierto sentido, responsable del conocimiento, la escuela se moldea como un espacio controlado, un tiempo dirigido y restringido. Así construye un tiempo activo y un tiempo pasivo del aprendizaje, haciendo de él un objeto manipulable y, por tanto, artificial. Ante ello surge la pregunta: ¿Puede el aprendizaje ser manipulado de tal forma que se experimente un on y un off como episodios procesuales? ¿Existe un "ambiente de desarrollo" que realmente favorezca el aprendizaje de los sujetos?

Vale la pena revisar estos cuestionamientos y otros más en favor de reconocer qué es esto que llamamos aprendizaje y de qué manera los haceres de los aprendientes están implicados en este proceso. Estas preguntas posiblemente ya tengan respuesta en variados programas de investigación en el ámbito de la educación, la psicología y especialmente las neurociencias aplicadas a la educación. Basta revisar algunas publicaciones para reconocer un paradigma común que relaciona objetivamente el medioambiente con el sujeto que lo habita, y que en su mayoría se encuentran ligadas a un principio tradicional aceptado a priori que valora el concepto de adaptación como un proceso de aprendizaje en que los individuos adquieren conocimientos tras la captación de estímulos del medio y adecuación de sus condiciones de acuerdo a tales constreñimientos. Lo que diferencia a estas propuestas está en la forma en que se plantea dicha adecuación. Por un lado encontramos aquellos que proponen una dependencia a los ordenamientos del entorno, mientras que otros apelan a la configuración de un programa de acción interno, un modo de plan predefinido. Lo que es común en estos programas es que tienen más o menos definida una idea de la percepción como clave para el aprendizaje, cuestión que no podemos negar. Sin embargo, mientras estos programas reducen la percepción a un problema lineal de input y output, en este trabajo se considera la perspectiva fundada en el enfoque enactivo que rechaza el modelo de una relación causal entre la intención y la acción (Di Paolo, 2015) con el propósito de orientarse hacia una abstracción diferente, en que la percepción no es algo que ocurre dentro de nosotros o algo que nos sucede, sino más bien una forma de apropiarnos y construir un mundo dentro de los patrones de contingencia de un entorno determinado (Noë, 2004). Este sentido de apropiación debe ser comprendido en la perspectiva de la creatividad, es decir, no nos apropiamos de un mundo preexistente por medio de la percepción, sino más bien lo hacemos emerger creándolo en nuestras interacciones con el espacio físico.

Se ha de aceptar que el aprendizaje no corresponde a una seguidilla de episodios independientes sino más bien a un fenómeno histórico, esto es, un proceso de transformaciones en el cual cada uno de los estados sucesivos surge como modificación de uno previo, nos encontramos, pues, ante una transformación causal y no ante un evento nuevo e independiente. Frente a esto ¿está siendo la escuela un espacio adecuado para el aprendizaje y qué tanto considera la experiencia encarnada de los aprendientes para favorecer estos procesos? 


\section{LA PERCEPCIÓN COMO PREMISA DEL APRENDIZAJE SITUADO}

La vida se los seres vivos en general, y los seres humanos en particular, es precisamente la de agentes situados cuya definición de acciones, como se ha dicho, no corresponde a la de un plan como potencialidad de alternativas contenidas en un repertorio posible ni tampoco a la de un acceso a informaciones contenidas en un mundo preexistente. La vida cotidiana, y en específico el mundo, no es una pista de aterrizaje en que nos arrojamos, y aunque los seres humanos llevemos adelante nuestras acciones encontrándonos permanentemente con actividades simultáneas en nuestros variados sistemas percepto-motores, estas acciones se definen como una realidad de acuerdo a su dependencia con la contingencia de las mismas. Como entidades cognitivas estamos estructuralmente ligadas a nuestro medio ambiente y nos relacionamos con él, de acuerdo a Varela, "desde la perspectiva establecida por la constante emergencia de características del agente mismo y en términos del papel que juegan tales redefiniciones en la coherencia del sistema" (2000: 67). El enfoque de la cognición situada, según Cosmelli e Ibáñez, "asume que el conocimiento está estructurado en el ambiente social, ecológico y físico que conforma la experiencia” (2007: 12).

Bajo esta perspectiva, rechazando los programas representacionistas y vitalistas, hablamos de la percepción como un problema situado y dependiente momento a momento de las contingencias operacionales del agente en cuestión. Los seres humanos tenemos un amplio repertorio conductual que nos permite llevar a cabo o emprender acciones concretas para cada episodio de nuestra experiencia fenoménica. ¿Corresponde esto acaso a un programa de acciones o a la determinación de nuestra conducta por parte de los estímulos del medio ambiente?, evidentemente no, de acuerdo a lo antes descrito, sería inadecuado pretender un operar bajo estos principios. La acción, antes entendida como consecuencia del estímulo, es el restablecimiento de una interacción con el entorno y sus constreñimientos de modo que el mundo emerge contingentemente a la acción y no preexiste (Moreno, 2014a), el acceso a la información contenida en un mundo externo y objetivo no tiene sustento operacional en los seres vivos.

Aun manteniendo la duda respecto al amplio repertorio conductual de los seres humanos, pueden surgir interrogantes respecto al origen de la acción efectiva, es decir, por qué las acciones emprendidas en un contexto particular parecen adecuadas a ese contexto, de modo que nos mantenemos estables en nuestra integridad, en otras palabras, por qué nuestras respuestas a los constreñimientos del entorno son adecuadas para conservar nuestra robustez como sistemas y nuestra identidad como unidades, o algo más cotidiano, por qué respondemos a una pregunta, cerramos los ojos ante una incandescencia, atendemos a un llamado y ejecutamos una orden. Parece ser que poseemos la capacidad de evaluar una situación dada interpretando sus exigencias y seleccionando una respuesta coherente, sin embargo estos quiebres en nuestra experiencia no tienen determinación ni origen objetivo, en cada uno de estos momentos de transición conductual emerge el sentido común como historia corpora ${ }^{1}$ y social, permitiéndonos de esta forma configurar un mundo (Varela, 2000). El conocimiento por sentido común no se puede encajonar en un conocimiento explícito y proposicional, más bien se trata de la disposición o conocimiento práctico acumulado por la experiencia vivida, equivalente al Know How ${ }^{1}$ descrito por Francisco Varela. Nuestra experiencia transcurre momento a momento, de una acción a otra que surge

Ver Varela, F. Ética y acción, 1996. 
con los propios recursos, estos goznes constituyen la base del carácter autónomo de nuestro conocer y, por tanto, de nuestra percepción-acción.

Los seres humanos percibimos guiando nuestras acciones en situaciones locales (Moreno, 2014a) que son cambiantes de manera contingente a nuestras acciones y dependientes del modo en que estamos encarnados, de acuerdo a Thompson "la percepción es la conciencia de las cosas en el mundo" (2005: 243), de todos modos no podemos quedarnos con la idea de que el mundo es un espacio trascendente que se nos presenta en su objetividad y mismidad, el mundo es, mejor dicho, una creación de nuestros estados perceptuales con la añadidura de nuestra experiencia de vida que nos permite significar nuestras interacciones. La percepción es inseparable de la acción, están unidas en un círculo virtuoso, es un principio de referencia en el que está involucrado el sistema nervioso. Thompson nos ejemplifica esta circularidad con la percepción del color explicando que "la percepción visual guía la acción y la acción dirige la percepción" (2005: 237), por su parte, Varela nos aclara que "no hay efecto del sistema nervioso (capacidad de movimiento, secreciones internas) que no tenga un efecto directo sobre una superficie sensorial" (2010: 258), esta advertencia es condición indispensable para el surgimiento de sistemas autónomos. En este sentido, la percepción se constituye como acción guiada perceptualmente y está permitida por la recurrencia de esquemas senso-motores como procesos autoorganizados de los cuales surgen las estructuras cognitivas. El concepto de estructuras cognitivas no debe ser reducido a un alcance fisiológico o anatómico pues está anclado a los detalles más minuciosos de nuestras experiencias de vida y es por esto que se afirma que tales estructuras definen el modo en que los seres humanos estamos encarnados. Se debe tener precaución también al momento de hablar de procesos mentales, cuando hablamos de los seres vivos apelando a lo mental o la conciencia, debemos hacerlo en el pleno reconocimiento de la experiencia vivida. Un problema fundamental de la neurociencia es que no puede quedar ajena a la búsqueda de respuestas para ese asunto. Por ello es clave la noción de conocimiento que emerge de esta abstracción de los sistemas cerrados, pues surge también al poner en evidencia la condición autónoma de los seres vivos en general.

Que nuestra cognición y las llamadas capacidades mentales estén encarnadas, significa que están envueltas en un mundo, un mundo que surge momento a momento de las acciones de los sujetos como agentes corporales que inscriben su identidad de manera activa en el hacer, y por tanto conocer. Un sistema autónomo produce su propio dominio de interacciones (Thompson, 2007), de todos modos la mera conducta es, en el mejor de los casos, una guía poco fiable de cómo son las cosas para una determinada persona (Noë, 2009). En el estado de vigilia, el mundo se presenta para nosotros de cierta manera y no de otra, en función de nuestros sentidos y capacidades cognitivas, que el mundo se presente o más bien se nos presente de esta forma quiere decir que nuestra conciencia revela el mundo y lo aprehende en estas formas (Thompson, 2015). A consecuencia de esto, reducir la interpretación del mundo de una persona a su manifestación conductual sería inadecuado si de lo que se trata es de abrir espacios de aprendizaje.

El mundo es percibido como tal en la medida en que nos movemos en él, y aunque estamos acostumbrados a creer que el mundo siempre está ahí esperando ser percibido, lo adecuado sería decir que el mundo emerge como consecuencia de nuestra acción presente. Nos movemos en el espacio físico, sistema nervioso incluido, como un todo global sin que en la acción podamos descifrar una dualidad entre nuestro sentir íntimo y nuestra corporalidad, es por ello que estas distinciones de propiedad, como sentido de 
pertenencia, corresponden únicamente a un dominio discursivo en que nиеstro cuerpo y nиеstra intimidad aparecen como elemento reflexivo, sin que tengan presencia como tales en la experiencia. El presente de nuestra acción es el espacio-tiempo en que emprendemos nuestra existencia sin los límites de una corporalidad y una experiencia por separado. Estas identidades, somática y cognitiva, propias de los mamíferos, coexisten dando forma al organismo interactuante. Francisco Varela, expone esto en las siguientes palabras:

No podemos salir del mundo determinado por nuestro cuerpo y nuestro sistema nervioso. No existe otro mundo excepto el que experimentamos por medio de estos procesos, que son premisas para nosotros y hacen de nosotros lo que somos. Nos encontramos dentro de un dominio cognitivo del cual no podemos salir o decidir dónde comienza o cómo se crea (2000: 383).

Ahora bien, no podemos reducir la percepción-acción a una linealidad, en realidad estamos frente a un fenómeno circular en que se relacionan superficies sensoriales y efectoras formando una red de correlaciones de percepción-acción no solo moduladas por las células neuronales, sino además originadas por ellas. Lo importante del cambio de las situaciones locales involucradas en la percepción es que está gatillado por la actividad del perceptor y no por el entorno, en este sentido, podemos dejar de lado la tesis representacionista y computacionalista del procesamiento de información para abrir paso a la estructura senso-motora del sujeto, en consideración de las identidades cognitiva y somática que, en definitiva, nos ayudan a conducirnos en la trama de los modos en que los individuos están encarnados. Estos modos, es decir, la forma en que los sujetos están encarnados, determinan las posibilidades de acción, lo que quiere decir que nuestra experiencia de vida es el factor principal para comprender la acción. Empero, cualquier interrupción en las correlaciones sensomotoras perturba la forma en que el perceptor experimenta el mundo (Gangopadhyay \& Kiverstein, 2009).

Si nuestras acciones nos permiten construir un mundo y dependen del modo en que estamos encarnados, entonces el significado de este mundo, a priori entendido como propio de él, también depende de ello. Es lógico que no podemos caminar en línea recta permanentemente, por las características físicas del espacio, en algún momento nos enfrentaríamos a un obstáculo que no sería dificil de identificar; por otra parte, cuando reconocemos un olor en el ambiente casi instantaneamente dirigimos nuestra mirada, lo mismo hacemos cuando escuchamos alguna alerta auditiva. De esto surge la pregunta ¿Por qué percibimos el mundo como si tuviese un significado dado? En las configuraciones de nuestras interacciones con el espacio físico se generan perspectivas o significados que permiten que se lleve a cabo una interacción viable, sin hablar de información, esto corresponde a que los agentes cognitivos no tienen una dirección externa, por el contrario existen en una condición de autonomía en que de manera activa generan y sostienen una identidad $^{2}$ constituida como punto de referencia para un dominio de interacciones.

Los profesores se mueven cotidianamente en principios y reglas pedagógicas, pudiendo también usar significados desde el punto de vista de los alumnos. Una metodología específica es estudiada y practicada por sus efectos en los estudiantes, sus aprendizajes, comprotamiento y disciplina. Pero, por otra vereda, la metodología y el aprendizaje son

Para este trabajo la idea de identidad se corresponde a un proceso y no a una descripción, lejos de ser un concepto personalista se trata de una coherencia del agente cognitivo que como proceso produce identidad, he ahí el cierre operacional y la noción de circularidad. 
meritorios de ser estudiados en tanto implican a la generalidad del alumnado, el efecto concreto de una metodología como facilitadora de un aprendizaje específico es factible por la identidad del alumnado en general. La metodología carece de significado a no ser que los estudiantes la vivencien como parte de su experiencia envueltos en ella en la continua producción de identidad. A los ojos del profesor, como observador, la metodología puede constituir un estímulo particular para gatillar ciertos comportamientos.

Varela propone una diferenciación entre medio ambiente y mundo que no es trivial y nos permitirá comprender de mejor manera el proceso de construcción de significado como contingente a la producción activa de identidad.

\footnotetext{
Me refiero a la distinción entre el medioambiente de un sistema de vida tal como se presenta al observador y sin referencia a una unidad autónoma -lo que de ahora en adelante llamaremos simplemente el medioambiente- y el medioambiente para el sistema, que se define en el mismo movimiento que dio lugar a su identidad y que sólo puede existir en esa definición mutua, a partir de ahora, el mundo del sistema.

La diferencia entre medioambiente y mundo es el excedente de significación que acosa al entendimiento de la vida y del conocimiento y, a la vez, está en la base de cómo un sí mismo alcanza su individualidad (2000: 60).
}

Cada encuentro implica una postura como conducta (aprendidas en la co-construcción de la ontogenia, como también características compartidas filogenéticamente) en las interacciones que resultan significativas desde la propia identidad del sistema y de acuerdo a las posibilidades del entorno. Por ejemplo, a nivel celular podemos reconocer sitios en la retina que se activan solo si el estímulo luminoso supera los $400 \mathrm{~nm}$. Por otro lado, y aún más lógico, los seres humanos desarrollamos nuestro vivir en la superficie terrestre y no en las profundidades del mar. Permanentemente distinguimos lo que es relevante y lo que no de acuerdo a una perspectiva proporcionada desde la individualidad que se corresponde a las interacciones. El significado no está en el medio ambiente, emerge de nuestras interacciones, los agentes cognitivos tienen una perspectiva de la que el medio carece, y ese excedente de significado es llevado a cabo en el constante vivir.

Los seres humanos nos vamos construyendo como agentes autónomos donde el movimiento debe ser destacado en la emergencia de la cognición. El movimiento, como parte de nuestra experiencia, deviene de un proceso del sistema nervioso como red que permite la unión de la musculatura con diferentes superficies sensoriales. Los resultados de las correlaciones senso-motoras están permitidas por el acoplamiento de los movimientos a partir del influjo de diversas modulaciones sensoriales del sistema nervioso. Estos ciclos senso-motores son fundamentales para la condición autónoma de los agentes cognitivos. De acuerdo a González, a través de estos ciclos "emerge la co-determinación entre lo que siente el organismo del entorno, que depende directamente de la forma en la que se mueve y cómo se mueve lo que depende directamente de cómo se siente" (2013: 106).

\section{COGNICIÓN DE ALTO NIVEL Y CONOCIMIENTO ESCOLAR}

Varios de los principios hasta ahora descritos se extienden a las condiciones de todos los seres vivos en general, el objetivo de este apartado es concretar aquellas lógicas considerando como agentes cognitivos a los seres humanos, como seres culturales donde 
emerge la cognición de alto nivel. Si bien esta categoría proviene del cognitivismo clásico y tiende a ser potencialmente engañoso, no es objetivo de este texto tratar su origen y aplicación, solo se utilizará esta clasificación ${ }^{3}$ debido a su utilidad para el caso. Se ha de entender este concepto en consideración de los procesos de auto-conciencia, empatía, recuerdos, abstracciones, imaginación, etc., en contraste a la aparentemente simple percepción. Su alcance y entendimiento como procesos mentales están lejos de ser un fenómeno y corresponden más bien a una explicación en que como se ha mencionado anteriormente, emerge la identidad del agente cognitivo, para este caso, el de los seres humanos. Así, lo mental pasa a ser la explicación de aquellos fenómenos vinculados a la cognición corporizada y, por tanto, a la experiencia del sujeto.

Comprendida la construcción de significado desde la acción de los sujetos, es menester concretar estas nociones operacionales en lo que respecta a las relaciones escolares. Los vehículos de la percepción se distribuyen a través del cerebro, el cuerpo y el mundo (Gangopadhyay \& Kiverstein, 2009), un mundo construido de manera activa en el movimentarse y encontrarse con los otros en el lenguaje. La cognición se produce por el acto de moverse y modular activamente los estados de nuestra experiencia, estados que se manifiestan conjuntamente favoreciendo la condición autónoma del agente humano, cuya identidad está basada sobre configuraciones endógenas de actividad nerviosa (Varela \& Depraz, 2003) como patrones de autoorganización. Esta actividad endógena configura el mundo significativo por medio de procesos de imaginación, abstracción y subjetivación, modulada por el acoplamiento sensomotor pero no determinada por él.

Evidentemente sería un error reducir la emergencia de procesos globales del agente cognitivo a los constreñimientos senso-motores, debido a que los procesos cognitivos de alto nivel están constreñidos por variadas modalidades sensoriales retroalimentadas y coherentes. La actividad endógena y la construcción de significado como capacidad de regular la dinámica de estado en función de sus consecuencias virtuales para la conservación de una forma de vida, es una actividad autoorganizada en interacción constante debido a una intensa interconectividad de regiones por lo que los actos mentales se caracterizan por la participación simultánea de estas regiones topográfica y funcionalmente distintas (Varela \& Depraz, 2003). Toda esta integración que configura correlaciones, además constituye bucles de interacción con el medio ambiente para poder actuar coherentemente, es decir, lejos de estar reducidas las interacciones con el ambiente a un proceso lineal de estímulo y respuesta, existe una coordinación, sincronización y reciprocidad de muchas regiones del organismo para que se posibiliten las interacciones con el ambiente, hay una orquesta en que diversos instrumentos se sincronizan para crear una armonía final.

De acuerdo a Varela y Depraz (2003), el cerebro se refiere, sobre todo, a su propia actividad, ocupándose constantemente del mantenimiento y regulación de los procesos del organismo. Esta actividad endógena se basa en su amplia interconectividad, pero también se debe a que el cerebro, siendo parte del organismo, no cesa de su autorregulación. Incluso en una posible deprivación sensorial, es decir, en ausencia de cualquier insumo del entorno, se engendran niveles de actividad que constantemente dan lugar a patrones dinámicos. Un lugar importante dentro de estas actividades lo ocupa la imaginación, descrita por los mismos autores como una fuente inagotable de diversas dimensiones, la imaginación es una

El concepto de high-level proviene del modularismo Fodoriano, para mayor información ver Fodor, J. A. (1983). The Modularity of Mind: An Essay on Faculty Psychology. Cambridge: MIT Press. 
ausencia que evoca. Existe un amplio consenso en la investigación actual que concuerda en la idea de que la capacidad de producir y manipular objetos imaginarios puede ser naturalmente explicada como la movilización endógena de las mismas capacidades neuronales involucradas en la visión y cognición en general, requiriendo así también de la participación de la memoria, la anticipación, el movimiento y de forma muy importante, el lenguaje. De esta forma se manifiestan actividades cruzadas y coherentes, por ejemplo, una imagen mental puede ser producida por un recuerdo perceptivo, pero también a través de una descripción (Varela \& Depraz, 2003).

La simultaneidad de las correlaciones de actividad permanente no se da en un instante aislado del vivir de los sujetos, sino que ocurre momento a momento en el presente vivido de los individuos que se corresponde con un espacio-tiempo en que se configura activamente la identidad. El aprendizaje involucra un sinnúmero de elementos que suceden en la individualidad, como también factores a considerar desde patrones colectivos de actividad. Por ello es inadecuado pretender subyugar esta realidad bajo el pretexto de la unicidad del contenido. Siguiendo a Toro y Niebles, "lo que se aprende no es sólo aquello de lo que se trata la sesión, en el caso de la escuela, sino también y fundamentalmente, de cómo se trata la sesión" (2013: 273), resultado de una suerte de compromiso afectivo que los aprendientes asumen en la comunidad. En la escuela tradicional el aprendizaje se basa en contenidos aislados, desconociendo así que lo aprendido realmente no es un contenido sino un modo de convivencia, los estudiantes por su lado aprenden al profesor (Moreno, 2014b). Ante esta perspectiva no podemos disminuir el estudio del aprendizaje a un evento en que tiene sentido un solo contenido, tampoco debemos concebirlo como una fase secuencial lineal, el aprendizaje es un proceso histórico que ocurre en el aprendiente y, como tal, no sucede en eventos independientes sino que como un proceso de cambios, que aunque sucesivos, cada modificación surge como transformación de un estado previo, es un proceso epigénico. Ahora bien, al afirmar que no debemos reducir el estudio del aprendizaje a un solo contenido, no se pretende decir que debamos, de modo aritmético, agregar varios contenidos simultáneamente, contenidos en el contexto de aquello que en la escuela identificamos. De lo que se trata es que debemos ampliar nuestra perspectiva respecto a las dimensiones y tipos de actividades que en el sujeto tienen espacio, es decir, considerar el amplio abanico de elementos de la cognición de alto nivel posiblemente en actividad y correlación. Frente a una clase, los estudiante no pueden limitarse a escuchar al profesor, también escuchan a los demás, conjugan su imaginación, sus abstracciones, sus ideas y pensamientos, identifican y modulan posturas y movimientos corporales, etc. De este modo, solamente desde la perspectiva del estudiante se puede saber lo que para él es relevante y aquello que para él es indiferente. El profesor como observador únicamente podría identificar un comportamiento.

En neurociencias, la evidencia sugiere que para la comprensión de las bases neuronales de la conciencia y los actos cognitivos debemos considerar que estos emergen a partir de patrones dinámicos de actividad a través de múltiples bandas de frecuencia y no a partir de circuitos específicos o clases de neuronas (Thompson \& Varela, 2001). Debido a esto, el presente vivido de los aprendientes se da en una sincronía de actividades y no simplemente en la unión de atributos sensoriales, es decir, hay toda una integración de las dimensiones del acto cognitivo, incluyendo aquí la memoria, el tono emocional y la planeación motora. Al ser los seres humanos sujetos sociales, vivimos imbricados unos con otros en la continua realización de nuestro vivir dando paso al entramado de conversaciones en que nos desenvolvemos como integrantes y formadores de una red de relaciones. Este 
importante nexo entre los patrones de actividad dinámica de los humanos y su condición de agente situado debe permitirnos entender que a los ciclos de acoplamiento senso-motor entre el sujeto y el medio, sumado a sus ciclos de regulación corpórea, debe agregarse lo que podemos denominar "interacción intersubjetiva", que efectivamente corresponde al dominio donde surge el lenguaje.

La condición de agente situado se evidencia potentemente en el juego de los niños. Al observar a los niños jugar los vemos inmiscuidos en un contexto en el que despliegan su experiencia corpórea, la cual, sumada a la mediación social, aparece como un control de las normas de actividad sin la necesidad de una conducción externa, es decir, la experiencia guía el acto de juego que, a menudo, aparentemente carece de sentido. Es más, el juego muchas veces parece romper con los principios Piagetianos de asimilación, pues los niños parecen separarse de un posible significado (como algo que se presenta y exige) en las diversas situaciones en que se desenvuelve. Este desapego es una actividad encarnada en que el niño participa activamente en la creación de un mundo, poniendo en presencia lo ausente para un observador: aparecen sabores de lo que no hay, sonidos de lo que no está y antropomorfismos que permiten que la actividad sea divertida y cercana. En el juego los objetos comienzan a estar imbuidos de nuevos sentidos a través de la acción en un aparente engaño a la realidad del observador, pues el niño trae a la mano "significados alienados" en los cuales, mediado y guiado por su experiencia, se somete activamente, de lo contrario el juego carecería de un sentido "realista". En el juego se da una doble paradoja, primero el niño se somete a la imaginación en un amplio espacio de incertidumbre, pero una vez persuadido en su actividad construye un mundo en el que se mueve con seguridad y certeza. Por otro lado, la combinación de una situación concreta con este significado alienado es la libertad para propagar un espacio adecuado de acciones situadas. Esta doble paradoja favorece la capacidad para crear nuevas normas (no arbitrarias), restricciones y posibilidades. Diferentes materiales y espacios aparecen como contextos en la construcción de significado durante la formación de la fantasía en los infantes (Sato et al., 2006). En el juego y sus múltiples actividades transitorias se evidencia la autonomía de los niños, pues permiten desplegar nuevas capacidades decisionales y de creación de sentido en que se define momento a momento la identidad de los participantes, esto es, los niños en el juego afirman activamente su identidad a través de su propias acciones. Es menester agregar que el juego, como conjunto de acciones, favorece el que los involucrados generen coordinaciones en la relación de forma que se satisface el vínculo. Las consecuencias que tenga el juego como sentido social es otro posible asunto, sin embargo el juego en sí mismo es la satisfacción del vínculo de las relaciones entre los participantes.

Es importante destacar que la perspectiva del aprendizaje situado supone la de agentes activos en el sostenimiento de su identidad a través de sus transformaciones. Para esto el aprendizaje debe comprenderse como una regularidad que se estabiliza en el sistema en su correspondiente acoplamiento con un medio que lo posibilita. La condición de agente en los seres humanos implica estar dotado de la capacidad de regular el comportamiento interactivo en relación a cambios en las condiciones del medio, y su estudio, tal como se propone respecto al metabolismo, debe tener como punto de partida el del sistema y no el del conjunto de componentes o propiedades (Cornish-Bowden et al., 2004). Nuestras vidas sociales están pobladas por diferentes fenómenos cognitivos (Di Paolo \& De Jaegher, 2015) activos en nuestras acciones conjuntas, en el percibir, en el compromiso verbal y no verbal, en la creación de significado, la intimidad, la confianza, el secreto, el conflicto, la 
negociación, asimetrías, fijación de normas, etc., todas ellas filtradas por una presencia perceptiva (Di Paolo, 2014) configurada como sensibilidad a la coherencia virtual que rodea las acciones actuales, es decir, los aspectos reales de un compromiso agente-mundo en que el individuo en su plena corporeidad se encuentra en disposición a la viabilidad de su vivir presente. En el lenguaje, los seres humanos además, a condición de esta presencia perceptiva, podemos monitorear, evaluar, regular y organizar nuestra existencia, como coordinación de coordinaciones consensuales (Maturana, 1990, 1992) el lenguaje ocurre como una posibilidad de emprender un modo de vida idiosincráticamente corporizado de participación y construcción de sentido modulada mutuamente cuando dos o más agentes autónomos se involucran en un encuentro interactivo (Cuffari et al., 2014). En seres humanos la construcción de significado y el aprendizaje debe entenderse desde el lenguaje, y por tanto se da a través de una historia de coordinaciones.

En los encuentros dados en el lenguaje cada individuo será el ser humano que su historia configura en un proceso de epigénesis (Maturana \& Nisis de Rezepka, 2004), el sistema cambia las condiciones de su acoplamiento con el entorno para satisfacer alguna restricción o norma o lograr un objetivo (Cuffari et al. 2014), empero todo lo que le pasa surge en la transformación de la estructura inicial de manera contingente a la historia del vivir del individuo.

El aprendizaje se da en tanto se forme parte de un flujo de acciones, en este sentido es ineludible un cambio de concepto respecto a lo que cotidianamente se refiere a los aprendizajes. Hay cierta costumbre a hablar de los contenidos de aprendizaje como un programa organizado de saberes asociados a un requerimiento básico, sin embargo al rescatar las nociones antes mencionadas, la mirada se dirige a entender que el involucramiento en los haceres es per se un proceso de construcción de significado en que se sincronizan variadas actividades del individuo. El corpus de aprendizaje, por ello, no corresponde a un contenido particular sino al contexto en que se descifra creativamente el contenido. De esta forma las distancias emocionales como diferencias en los estados de posibles conductas asociadas en la interacción constituyen los modos de aprendizaje que experimentarán los aprendientes. A consecuencia de esto, sería adecuado hablar de aprendizajes escolares en el marco de un espacio-tiempo dinámico en vez de contenidos de aprendizaje bajo los pretextos clásicos.

Los modos en que los estudiantes se despliegan caracterizan las interacciones de su vivencia escolar en una trenza de estados con emociones y discursos inscritos en contextos particulares que configuran su presente vivido. La emoción, entendida como un modo de estar en la acción, está asociada al acto del estudiante, a su acción con sentido, teniendo el discurso un espacio en esta trama participando a modo de representación de un flujo de acciones que tienen asociadas un sentido emocional. Los discursos escolares especifican también los aprendizajes de los estudiantes construyendo un espacio simbólico compartido que media estados con estados. De acuerdo a Toro y Niebles (2013), existe un patrón de contingencias tanto normativas, espaciales como institucionales que condicionan el actuar de los estudiantes, estos patrones provocan en la escuela el extravío de su contexto haciendo que el proceso de enseñanza y el proceso de aprendizaje sucedan de manera diferente a como acontecen en la naturalidad del vivir de los estudiantes (Moreno, 2014b).

El despliegue que vivencian los aprendientes en la escuela constituye un pilar fundamental en el entendimiento de los procesos de aprendizaje, pues la acción creativa configura el corpus de conocimiento de los contextos escolares. Los espacios y tiempos que caracterizan a la escuela deben permitir diferentes grados de libertad de acuerdo a 
las exigencias propias de cada estudiante, más allá de un programa rígido que pretenda implementarse a modo de regla. La práctica cultural compartida que caracteriza al lenguaje solo puede ser aprendida por una persona entre muchas, dentro de un ecosistema cultural específico (Noë, 2009). Al requerir esta práctica un sustento emocional de socialidad, los grados de libertad que la escuela proporcione favorecerán los aprendizajes en un estado natural. Esta idea de estado natural el autor la atribuye al aprendizaje situado que se caracteriza por respetar las condiciones biológicas y culturales de los aprendientes, en contrapartida con un aprendizaje artificial que pretende forzar mecanismos y métodos a través de modelos memorísticos y represivos. En este sentido, el aprendizaje en estado natural se concibe como un proceso de flujo continuo donde ciertos estados conllevan a la estabilización de una regularidad en la acción en que el contenido de aprendizaje se construye como un mundo que tiene que ver con lo que pasa en la corporeidad, un mundo creado en la acción y que a la vez mueve a la acción de una manera concreta. Así el conjunto de actividades de los estudiantes genera y sostiene el espacio en que actúan, el curso de sus acciones define momento a momento su propia deriva.

Es preciso aclarar que las distinciones de corporalidad y actividad son solamente distinciones, es decir, en el sentido de la experiencia vivida soy en la acción del mismo modo en que soy en el cuerpo. El conocimiento depende de estar en un mundo inseparable de nuestro cuerpo, nuestro lenguaje y nuestra historia social, en síntesis, de nuestra corporización (Varela et al., 1997), por la misma parte, las emociones son determinantes en el conocimiento, o mejor dicho, en el aprendizaje, pues algunas gatillan y otras inhiben la acción, de modo que una emoción corresponde a una disposición a la acción.

Los niños son seres que aprenden y que además aprenden a vivir cualquier tipo de vida en que sucede que viven, en estas circunstancias, el tipo de ser humano que llegan a ser los niños al crecer surge como una identidad sistémica que ha sido conservada en la historia de interacciones (Maturana \& Nisis de Rezepka, 2004). Esto es, la escuela cumple un rol fundamental en la formación de seres humanos, el tipo de relaciones que se generen en el vivir en la escuela, dependiente de la emocionalidad que ahí se geste, constituye el modo en que aprenderán los involucrados y el modelo de configuración de un tipo de ser humano. Cuando los espacios y tiempos de la escuela se formulan a favor de estructurar un futuro estudiante, se niega rotundamente la identidad del educando rechazando a la vez los mundos que surgen en el vivir propio de cada uno de ellos. La escuela, por tanto, como un espacio-tiempo contribuye a los procesos educativos de dos modos, en primer lugar al crecimiento de un tipo particular de ser humano, coherente a su modo de vida, y por otro lado en el desarrollo de habilidades operacionales concretas de cada ámbito del vivir. Cuando estos dos modos se permiten en respeto a la conservación de una identidad biológica y cultural del educando, el aprendizaje ocurre en estado natural.

\section{LA ESCUELA, UN ESPACIO-TIEMPO PARTICULAR}

El ámbito de aprendizaje de los seres humanos está lejos de ser acotado, en el lenguaje ampliamos el campo de coordinaciones de modo que las interacciones y los discursos median ciertos comportamientos que generan la coordinación de la conducta. En sistemas que aprenden, es decir, sistemas que tienen conducta plástica, las interacciones sociales que median las coordinaciones conductuales son aprendidas en la misma coordinación de 
conductas, es por ello que el contexto y la emocionalidad en que se dan las coordinaciones conductuales constituye el fundamento del corpus de conocimiento que se genera. Es así como en la escuela no se aprenden contenidos, sino los contextos en que surgen las relaciones que dan paso a la aparición de los contenidos. El educador configura un espacio de convivencia para la transformación de los participantes de las relaciones que en la escuela se producen, los estudiantes aprenden al profesor en sus formas relacionales y se transforman en la convivencia según el convivir que ellos viven con los adultos con quienes conviven (Maturana \& Vignolo, 2001). Las personas y los niños en particular propenden a aprender (Calvo, 2012; Moreno et al., 2013), de modo que la forma en que los adultos hacen uso inteligente de su entorno, su ecología, influye de manera sustancial en el desarrollo intelectual, social y físico del niño (Lebeer, 2013). Esta predisposición innata al aprendizaje no se corresponde con un contenido específico, sino simplemente al aprendizaje. Los seres humanos pueden describirse como especialistas en aprendizaje no especializado (Varela \& Hayward, 1997), siendo esta una orientación para comprender que al ser sujetos autónomos, son ellos mismos el referente para lo adecuado y lo no adecuado de su conducta. El mundo, o bien dicho, los mundos, están moldeados en función de nuestras propias expectativas, nuestra historia y nuestro desarrollo, por lo que la imagen de ese mundo es inseparable de cada cerebro particular, de cada persona.

No puede llamarnos la atención que la escuela tradicional plantee abiertamente como su misión, formar jóvenes competentes y capaces de adaptarse a un mundo particular. Una visión centrada en el futuro que propone sujetos que puedan responder a las exigencias, principalmente, del mercado y la productividad. Las exigencias y las expectativas que la escuela impone son negadoras de la autonomía (Maturana \& Vignolo, 2001), a la vez que construye su corpus de conocimiento propio como conductas efectivas en el ámbito determinado de la escuela, dando cabida a sus propias explicaciones y normas, este corpus como contenido va más allá de aspectos teóricos expresados en un programa: es coherente a la formulación de una pauta de comportamientos que vienen a caracterizar una particular configuración espacio-tiempo, esto es, la escuela es una real red de conversaciones en que sus participantes conviven como miembros de una cultura específica.

Hay dos trampas que amenazan la educación. Por un lado y vinculada a lo descrito antes, la idea de que el mundo de las cosas da instrucciones al organismo se modela como un sostén epistemológico que encabeza los planteamientos pedagógicos y perpetúa una jerarquización de las relaciones y una linealidad de los discursos e intenciones atribuyendo principios semánticos a los fenómenos de aprendizaje donde aparecen ideas como entradas y salidas respecto a mensajes, flujos de información y comportamiento. La intención de este trabajo es coordinar una mirada que comprenda estas entradas como perturbaciones en la dinámica intrínseca del educando, en lugar de instrucciones a seguir, y los estados internos como compensaciones autoorganizadas provocadas por tales perturbaciones, más que como representaciones de estados externos de ciertos eventos. Esto es, se requiere de una visión del ser humano como fuente de su propia actividad, un enfoque que lo conciba como un agente que especifica sus propios dominios de interacción y no como un transductor cuya función es transformar una instrucción en una respuesta específica. Bich (2012), aclara que la autonomía es en un sentido la capacidad para responder a las perturbaciones externas a través de variaciones autodeterminadas (autonomía externa), y por otro la autoproducción, automantención y autodistinción del medio ambiente a través del control activo de sus procesos de transformación (autonomía interna). A este paso, 
en la escuela se requiere de ambientes activos desafiantes para potenciar y fortalecer sus capacidades cognitivas, emocionales, motoras, éticas y estéticas (Calvo, 2013), teniendo como punto de partida la premisa fundamental de la cognición humana, es decir, saber que esta se estructura desde una realidad encarnada, a saber, como contexto de mecanismos cognitivos y como estructura experiencial.

Por otra parte, la segunda trampa que amenaza a la educación es la permanente intención por controlar, incluso predictivamente, todos los procesos escolares, suponiendo que ante la ausencia de un control y la emergencia de la incertidumbre el resultado sería inevitablemente un caos arbitrario. Sin embargo, mientras el espacio formal de la escuela intenta prevalecer, la informalidad naturalmente ejerce una presión constante. En este sentido, el proceso de enseñanza y el proceso de aprendizaje, en lo que conocemos como educación informal, suceden de manera diferente a como acontecen en el contexto escolar tradicional (Moreno, 2014b), de tal manera los procesos informales de la escuela parecen no seguir una secuencia lineal y en cierta medida están naturalmente más abiertos a las relaciones sinérgicas y divergentes.

Sumado a lo anterior, es imprescindible agregar que en la escuela como espacio-tiempo las acciones no suceden como actos aislados, el sentido de la acción, más allá del despliegue y emprendimiento de la propia existencia (Toro, 2012), está cubierto de un aliento social, con afectos y disposiciones que alimentan el corpus de conocimiento escolar agregado a los principios formales del contenido curricular. La divergencia y diversidad de los despliegues corpóreos, como también las formulaciones discursivas que emergen de las relaciones escolares es propia del sentido y presencia de cada sujeto en su experiencia vivencial, de esto se desprende que los intentos por homogenizar las personalidades a modo de modelo institucional y cultural constituyen una ceguera frente a la autonomía de los individuos. El desafío tiende a esclarecerse, la acción que se emprende por parte de la agencia cognitiva contiene intrínsecamente una intencionalidad que operacionaliza el acto. Ahora comienza a comprenderse en concretitud la valencia del excedente de significado como la madre de la intencionalidad (Varela, 1992), presente en las continuas actividades paralelas que el sujeto vivencia, esto es, el fundamento de la condición situada de la existencia humana está justamente en la perspectiva de la acción, construcción encarnada desde la experiencia y actitudes en la ontogenia individual. Los tiempos y espacios escolares son referidos por los estudiantes en relación a esta perspectiva establecida por la emergencia constante de sus propiedades en términos del rol que semejante distinción en marcha juega en su propia coherencia. Es así como las imposiciones de la escuela pueden descontextualizar los estados de los estudiantes, ubicándolos en realidades impropias y en cierta medida alienadas que desestabilizan el sentir del acoplamiento.

\section{APUNTES FINALES}

Sin lugar a dudas, orientar la mirada a la perspectiva descrita supone la adquisición de diferentes desafíos. Este trabajo ha buscado servir como apoyo y empuje a posibles investigaciones que busquen un acercamiento a la cotidianidad de las relaciones escolares. Para ello se proponen diferentes nociones a considerar:

El fenómeno del conocer está lejos de ser, en términos operacionales, la captación o representación interna de antecedentes objetivos de una realidad absoluta. Las interacciones 
que un individuo establece con el medio están mediadas por el operar del sistema nervioso como sistema cerrado de relaciones cambiantes de actividad. El cambio que en el dominio de estados posibles el sistema nervioso puede adoptar constituye el aprendizaje, teniendo lugar a lo largo de la ontogenia del individuo como resultado de sus interacciones.

El vivir humano ocurre precisamente como un vivir de agentes situados en que el conocimiento tiene una estructuración ecológica histórica desde las experiencias corpóreas vividas de los individuos. Este vivir es una coparticipación en las vidas individuales donde cambian juntos de manera coherente al flujo de interacciones dentro del lenguaje. Nuestra cognición está encarnada, esto es, está envuelta en un mundo que surge desde las propias acciones del sujeto.

Las acciones de los individuos inscriben activamente una identidad en la continua generación de su propio dominio de interacciones. Los encuentros con el medio están caracterizados por la significación de la interacción, elemento que emerge desde cada discontinuidad, vista como el comienzo de una acción sobre lo que falta en la parte del individuo que conserva su identidad cuya naturaleza surge a través de un proceso distribuido. La continua creación de significado supone el rechazo a las ideas representacionistas de la cognición y aquellas tendientes a homologar al individuo con un sistema de computación a través de concepciones como procesamiento de información y transmisión de mensaje.

La escuela se constituye como un espacio-tiempo dinámico. Sin embargo, este dinamismo está sujeto a ciertas interpretaciones. Podemos decir que los intentos de formalidad de la escuela promueven formulaciones estáticas que en cierta manera hacen perdurar modelos relacionales específicos a la vez que ciertas direccionalidades internas tienden a la divergencia de interacciones posibles. Partiendo de la premisa de nuestra experiencia como distinciones sobre los rasgos de la vida, podemos referirnos a un multiverso de realidades que conforman la experiencia vivida, esto en la escuela está atravesado por una permanente creación de conocimiento, que en un contexto cultural particular constituye un corpus propio que define conductas efectivas en su ámbito determinado. El corpus de conocimiento de la escuela, el conocimiento escolar, sobrepasa los límites que puedan imponer ciertos contenidos curriculares abarcando ampliamente una configuración espacio-tiempo que caracteriza una red de conversaciones como cultura interna. El corpus de aprendizaje no corresponde a un contenido particular sino al contexto en que se descifra creativamente el contenido. El despliegue vivencial de los aprendientes no ocurre aislado sino que está envuelto en un contexto, el contexto escolar que configura disposiciones en las acciones de la comunidad.

Finalmente, uno de los puntos trascendentales para este trabajo es el de la comprensión de la condición de autonomía en que operan los seres humanos. Se ha descrito la autonomía como la generación y sostenimiento activo de una identidad, de manera que se conforma como un punto de referencia para un dominio de interacciones. Los desafíos de la escuela son claros en este sentido: permitir en la creación de relaciones un despliegue pleno de la autonomía de sus participantes. Los docentes podrán cumplir un rol mediador no en tanto se inserten metafóricamente en un plano intermedio entre el contenido y el aprendiz, sino que en la medida en que actúen como facilitadores en la creación de relaciones que establece el educando con el entorno, esto en el pleno respeto de su autonomía biológica, en otras palabras, ser un mediador es constituirse como un hacedor social que requiere del estudio permanente del corpus de conocimiento escolar que se configura en el espaciotiempo. 


\section{REFERENCIAS BIBIOGRÁFICAS}

Bich, L. (2012). L'ordine invisibile. Organizzazione, autonomia e complessità del vivente. Soveria Mannelli: Rubbetino Università.

Calvo, C. (2012). Del mapa escolar al territorio educativo. La Serena: Universidad de la Serena.

Calvo, C. (2013). ¿Inclusión escolar o educativa: Dilema o Paradoja? En S. López De Maturana, Inclusión en la vida y la escuela. Pedagogía con sentido humano (pp. 48-67). La Serena: Universidad de La Serena.

Cornish-Bowden, A., Cárdenas, M., Letelier, J.-C., Soto-Andrade, J., \& Guíñez, F. (2004). Understanding the parts in terms of the whole. Biology of the Cell, 96, 713-717. doi:10.1016/j. biolcel.2004.06.006

Cosmelli, D., \& Ibáñez, A. (2007). Dinámica, intencionalidad y corporeización de la mente. Pasos hacia la comprensión de los límites y posibilidades de los nuevos enfoques de la cognición. En A. Ibáñez, \& D. Cosmelli, Nuevos enfoques de la cognición (pp. 9-25). Santiago: Universidad Diego Portales.

Cuffari, E., Di Paolo, E., \& De Jaegher, H. (2014). From participatory sense-making to language: there and back again. Phenomenology and the Cognitive Sciences. doi:10.1007/s11097-014-9404-9

Di Paolo, E. (2014). The worldly constituents of perceptual presence. Frontiers of Phycology, (5), 1-2. doi:10.3389/fpsyg.2014.00450

Di Paolo, E. (2015). Interactive Time-Travel: On the Intersubjective Retro-modulation of Intentions. Journal of Consciousness Studies, 22 (1-2), 49-74.

Di Paolo, E., \& De Jaegher, H. (2015). Toward an embodied science of intersubjectivity: widening the scope of social understanding research. Frontiers in Psychology, (6), 1-2. doi:10.3389/ fpsyg.2015.00234

Gangopadhyay, N., \& Kiverstein, J. (2009). Enactivism and the Unity of Perception and Action. Topoi, 28 (1), 63-73.

González, X. (2013). La auto-producción de la subjetividad: autopiesis y cognición corporizada de alto nivel. En P. Razeto, \& R. Ramos, Autopoiesis. Un concepto vivo (pp. 95-118). Santiago: Universitas Nueva Civilización.

Lebeer, J. (2013). La modificabilidad, neuroplasticidad y la ecología del aprendizaje en función de la educación inclusiva. En S. López De Maturana, Inclusión en la vida y la escuela. Pedagogía con sentido humano (pp. 224-247). La Serena: Universidad de La Serena.

Maturana, H. (1990). Emociones y Lenguaje en Educación y Política. Santiago: Hachette-CED.

Maturana, H. (1992). El sentido de lo humano. Santiago: Ediciones Pedagógicas Chilenas S.A.

Maturana, H., \& Nisis de Rezepka, S. (2004). Transformación en la convivencia. Santiago: J.C Sáez.

Maturana, H., \& Vignolo, C. (2001). Conversando sobre educación. Perspectivas, 4(2), 249-266.

Moreno, A., Calvo, C., \& López De Maturana, S. (2013). Aprender en y desde la motricidad humana: Educación, escuela y mediación pedagógica. Revista Iberoamericana de Educación, 62, 203-216.

Moreno, B. (2014a). Acción y cognición: una educación física de la acción presente. Educación Física y Ciencia, 16(2). Recuperado desde http://www.efyc.fahce.unlp.edu.ar/article/view/ EFyCv16n02a04/

Moreno, B. (2014b). Desescolarización de la Escuela: Hacia el rescate de lo educativo. En R. Arnt, G. Canihuante, B. Moreno, P. Pereira, L. Razeto, P. Razeto, H. Richards, A. Valdebenito. \& C. Valenzuela, La Escuela y los Ambientes y Contextos de la Enseñanza-Aprendizaje (pp. 149-184). Santiago: Universitas Nueva Civilización.

Noë, A. (2004). Action in perception. Massachusetts: Mit Press.

Noë, A. (2009). Out of our heads: Why you are not your brain, and other lessons from the biology of consciousness. New York: D\&M. 
Sato, K., Kashima, M., Hosaka, K., \& Nagahashi, S. (2006). Emergency of creativity in childrens's play fantasies and world-making. Research ans clinical center for child development, 28 (1), 51-61.

Thompson, E. (2005). Colour Vision: A study in cognitive science and philosophy of perception. New York: Routledge.

Thompson, E. (2007). Mind in life: Biology, phenomenology, and the sciences of mind. Massachusetts: Harvard University Press.

Thompson, E. (2015). Waking, Dreaming, Being. New York: Columbia University Press.

Thompson, E., \& Varela, F. (2001). Radical embodiment: neural dynamics and consciousness. TRENDS in Cognitive Sciences, 5(10), 418-425.

Toro, S. (2012). Desde la acción a la enacción. Más allá del movimiento y de la educación Física. Estudios Pedagógicos, 38 (1), 211-230.

Toro, S., \& Niebles, Á. (2013). Corporeidad y aprendizaje en el contexto de la Enseñanza General Básica: comprensión y descripción de los procesos de construcción de conocimiento desde la acción relacional de los actores. Estudios Pedagógicos, 39(1), 269-284.

Varela, F. (2000). El fenómeno de la vida. Santiago: Dolmen.

Varela, F. (1992). Autopoiesis and a biology of intentionality. En B. McMullin, \& N. Murphy (Eds.), Autopoiesis and perception (pp. 1-14). Dublin: School of Electronic Engineering Technical Report.

Varela, F. (1996). Ética y Acción. Santiago: Dolmen.

Varela, F. (2010). El círculo creativo. Esbozo historiconatural de la reflexividad. En P. Watzlawick, La realidad inventada (pp. 251-263). Barcelona: Gedisa.

Varela, F., \& Depraz, N. (2003). Imagining: Embodiment, Phenomenology, and Transformation. En A. Wallace, Buddhism and Science: Breaking New Ground (pp. 195-226). New York: Columbia University Press.

Varela, F., \& Hayward, J. (1997). Un puente para dos miradas. Santiago: Dolmen.

Varela, F., Thompson, E., \& Rosch, E. (1997). De cuerpo presente. Barcelona: Gedisa. 